\title{
Carbohydrate chemistry in California
}

By combining an improved oligosaccharide synthetic route with protein-binding and cell-growth assays, Caltech researchers can investigate the biological roles of specific glycosaminoglycan sulfation patterns.

To Atkins dieters everywhere, carbohydrates are the enemy. To a lot of chemical biologists, however, carbohydrates are pretty neat.

Carbohydrates in the form of oligosaccharide and polysaccharide sequences are crucial protein modifications involved in countless biological functions, from development to immune response to cell-cell communication. Studying protein glycosylation is a challenging pursuit because of the complex structures of carbohydrates. Furthermore, they are difficult to probe via genetic intervention because glycosylation is a nontemplated post-translational process. Fortunately, a variety of tools are rapidly being generated, as researchers have begun to recognize the biological and medical importance of glycosylation (see Box 1).

California Institute of Technology professor Linda Hsieh-Wilson and her colleagues are interested in one such class of carbohydrates known as the glycosaminoglycans, or GAGs for short, and their roles in neuronal development and regeneration. GAGs are often sulfated in different patterns, which have been implicated as encoding important functional information for various biological processes. "The complex chemical structures of GAGs provide instructions for protein binding and regulation," explains Hsieh-Wilson.

Until now, however, a molecular-level understanding of these sulfation sequences was missing, mainly because the chemical synthesis of GAGs is extremely challenging. The first step Hsieh-Wilson and her colleagues took was to design a streamlined synthetic route to making tetrasaccharide chondroitin sulfate glycosaminoglycans with varied sulfation patterns. "I had two or three people on the project for four years, with the goal being not only to make one molecule but to make a variety of different molecules where we control the sulfation pattern," says Hsieh-Wilson.

With chondroitin sulfate sequences in hand, the researchers immobilized the molecules on a microarray to investigate their interaction with the growth factor midkine, which is involved in the development and repair of neural tissue. Midkine bound strongly to one of the sulfation sequences, CS-E. Furthermore, they discovered that brain-derived neurotrophic factor, which is involved in regulation of nervous system development, also had a strong preference for CS-E.

Next they examined the effects of sulfation patterns on primary hippocampal neuron cell growth. Overwhelmingly, they found that CS-E was required for neurite outgrowth on coverslips coated with the molecule, whereas cell growth did not occur on surfaces coated with any of the other sequences. "Even a closely related analog with the same structure and overall charge had no effect, which suggests that the interactions between proteins and glycosaminoglycans can be extremely selective," says Hsieh-Wilson. CS-E also stimulated the outgrowth of several other neuronal cell types and its activity was inhibited by blocking the receptors for midkine and brainderived neurotrophic factor, suggesting that this sequence may recruit growth factors and activate downstream signaling pathways.

These results led the researchers to hypothesize the existence of a precise 'sulfation code' on the backbone of chondroitin sulfate. "It's been challenging to probe these molecular interactions using just biological approaches alone," remarks Hsieh-Wilson. "Synthetic organic chemistry can provide unique insights and, when used with a battery of other biological techniques, you can start to address some really interesting questions."

\section{Allison Doerr}

\section{RESEARCH PAPERS}

Gama, C.I. et al. Sulfation patterns of

glycosaminoglycans encode molecular recognition and activity. Nat. Chem. Biol. 2, 467-473 (2006).

\section{BOX 1 CHEMICAL REPORTERS OF FUCOSYLATION}

The chemical labeling of another type of polysaccharide post-translational modification, the fucosylated glycan, is the subject of two recent reports led by Chi-Huey Wong of the Scripps Research Institute (Sawa et al., 2006) and Carolyn Bertozzi of University of California,

Berkeley (Rabuka et al., 2006).

Fucose is often an important component of glycans involved in cellcell interactions and cell migration. It is also known to be particularly prevalent in cancer tissues, so there is a great deal of interest in investigating fucosylated antigens as potential cancer biomarkers.

Wong's and Bertozzi's laboratories have devised similar labeling strategies by harnessing the fucose 'salvage' metabolic pathway to incorporate a bio-orthogonal chemical handle. Enzymes in this pathway tolerate and incorporate azido-modified fucose analogs into polysaccharides. Using either the Staudinger ligation or 'click' chemistry, both groups selectively labeled azidofucose on the cell surface and then stained the cells with a fluorescent probe. Additionally, Wong's group showed that fucosylated glycans could be imaged inside cells by directly labeling azidofucose with a new fluorogenic naphthalimide-based molecule bearing an alkyne handle.

\section{RESEARCH PAPERS}

Sawa, M. et al. Glycoproteomic probes for fluorescent imaging of fucosylated glycans in vivo. Proc. Natl. Acad. Sci. USA 103, 12371-12376 (2006).

Rabuka, D. et al. A chemical reporter strategy to probe glycoprotein fucosylation. J. Am. Chem. Soc.; published online 23 August 2006. 